\title{
First measurements of the temporal evolution of the plasma density in HiPIMS discharges using $\mathrm{THz}$ time domain spectroscopy
}

\author{
Steffen M. Meier ${ }^{1}$, Ante Hecimovic ${ }^{2,3}$, Tsanko V. Tsankov ${ }^{1}$, \\ Dirk Luggenhölscher ${ }^{1}$, Uwe Czarnetzki ${ }^{1}$ \\ E-mail: Tsanko.Tsankov@rub.de \\ ${ }^{1}$ Chair for Plasma and Atomic Physics, Ruhr-University Bochum, Germany \\ ${ }^{2}$ Chair for Experimental Physics II - Reactive Plasmas, Ruhr-University Bochum, \\ Germany \\ ${ }^{3}$ Max-Planck-Institut for Plasma Physics, Boltzmannstraße 2, 85748 Garching, \\ Germany
}

\begin{abstract}
In this paper the novel technique of $\mathrm{THz}$ time domain spectroscopy has been applied to obtain time-resolved measurements of the plasma density in the active zone of a HiPIMS discharge with a titanium target. The obtained peak values are in the range of $10^{12}-10^{13} \mathrm{~cm}^{-3}$ for discharge current densities of 1 to $4 \mathrm{~A} / \mathrm{cm}^{2}$ at 0.5 and $2 \mathrm{~Pa}$ argon pressure. The measured densities show good correlation with the discharge current and voltage and the intensity of various atomic and ionic lines. The well known phases of the discharge have been identified and related to the variation of the electron density. The measurement results show that the plasma density remains nearly constant during the runaway/self-sputtering phase. Based on that it is conjectured that singly charged titanium ions are the dominant ion species during this phase.
\end{abstract}

PACS numbers: 52.55.-s, 52.70.-m, 52.30.-q, 52.35.-g

Submitted to: Plasma Sources Sci. Technol. 


\section{Introduction}

Magnetron sputtering is a physical vapour deposition (PVD) technique used for deposition of functional thin films on various substrates. High power impulse magnetron sputtering (HiPIMS) constitutes a subclass of the magnetron sputtering method in which the plasma is generated in short pulses, typically $10-100 \mu \mathrm{s}$, with a low duty cycle that is commonly about $1 \%$. The use of a pulsed plasma with a low duty cycle is motivated by the possibility to use high power densities during the pulse in order to achieve a high plasma density with high portion of metal ions, while preventing the target from melting $[1,2]$. These features constitute the foremost advantages of the HiPIMS process over DC magnetron sputtering or pulsed DC magnetron sputtering [3]. Furthermore, high electron density correlates with a high ionization degree of the sputtered material. The latter is desired, as it allows the energy of the ions hitting the substrate to be tuned by using a bias voltage. The energy of the ions is directly related to the densification of the deposited films [4]. Choosing the correct energy provides the desired film structure according to the structure zone diagram [5].

The high sputter and deposition rates make the diagnostic characterization of these plasmas a challenge. One of the most common diagnostics methods used is optical emission spectroscopy (OES). It provides information on the density of the emitting species convoluted with the energy distribution function of the electrons. Typical results of time resolved OES measurements in HiPIMS plasmas demonstrate a fast increase in the emission of the Ar atoms at the beginning of the pulse. The emission then either saturates or decreases in intensity. The latter occurs at high current densities that lead to significant sputtering of the target material. This sputtered material can then displace the argon particles from the vicinity of the target leading to argon gas rarefaction. The spectrum during the later phase of the discharge pulse is typically dominated by emission from Ar ions and from species of the sputtered material, both atoms and ions [6-9]. However, the temporally varying species composition of the plasma during the pulse makes it challenging to extract any further information on the plasma through collisional-radiative models. Therefore, the plasma density in HiPIMS plasma is typically measured using a Langmuir probe.

The Langmuir probe measurements are performed in the volume between the cathode/target and the substrate [10-14]. In the substrate vicinity, these measurements are quite sufficient. However, the measurements of the electron density in the target vicinity are quite challenging. Firstly, the Langmuir probe strongly perturbs the plasma as it intersects the closed magnetic filed lines and drains the electrons from the plasma. The magnetic field itself and the deposition of the sputtered material onto the probe tip and its holder pose further complications for the probe measurements. In HiPIMS discharges the high plasma density can easily lead to overheating and melting of the probe. The non-stationarity of the processes and the necessity of temporally resolved measurements further add to the complexity of the problem.

Measurements with the Langmuir probe in the volume outside the magnetic 
confinement zone (down to $2 \mathrm{~cm}$ away from the target surface) show that the typical electron density is of the order of $10^{12}-10^{13} \mathrm{~cm}^{-3}$ [10-14]. The modelling results indicate densities in the same range of about $10^{13} \mathrm{~cm}^{-3}[15,16]$. Time resolved measurements show an increase of the electron density during the pulse. The peak density is reached either during the pulse or shortly after the pulse end, depending on the distance from the target [10-12]. The contribution from Bohlmark et al [17] corroborates the correlation between the position and the peak electron density by showing a $2 \mathrm{D}$ evolution of the plasma density propagation away from the target in the afterglow. Indirect measurements of the plasma density have been recently performed using either a triple probe at the edge of the closed magnetic field lines [18], or pins in the target [19]. The obtained densities are in the order of $10^{13} \mathrm{~cm}^{-3}$, exhibiting a strong modulation due to the presence of spokes in HiPIMS plasmas [20].

The energy distribution of the electrons in a HiPIMS plasma has been also obtained, both experimentally [21] and by modelling [22]. It has been found to be predominantly Maxwellian with a high energy tail from about $30 \mathrm{eV}$ to the energy corresponding to the value of the cathode potential. This energetic tail is due to secondary electrons released from the target and accelerated in the high-voltage sheath in front of it. These electrons account for up to $10 \%$ of the total electron density [23,24]. Beside acceleration of secondary electrons in the sheath, electrons with sufficient energy can be obtained also through Ohmic heating of the current carrying electrons in an extended presheath [25]. The energetic electrons are the main contributor to the ionization in the plasma and the reason for the observed ionic lines.

Despite these exhaustive measurements, direct experimental information on the temporal evolution of the plasma density in the vicinity of the target, i.e. in the ionization zone is lacking in the literature. In this paper we use $\mathrm{THz}$ time domain spectroscopy (TDS) to experimentally obtain for the first time this important quantity. This diagnostic technique is chosen due to its non-invasive character, good temporal and spatial resolution and its extreme suitability for dense plasmas (densities beyond $\left.10^{12} \mathrm{~cm}^{-3}\right)$. The measurements are performed in a HiPIMS discharge, operating in argon with a titanium target. The time resolved measurements of the plasma density are complemented with optical emission spectroscopy data. The latter allows not only to observe the temporal evolution of the density of the emitting species from the plasma but also provides a reference for comparison with other investigations where often optical measurements are a key diagnostic tool. The correlation of the temporal evolution of the discharge voltage and current, the plasma density and the emission intensity from various atomic and ionic species shows the well known phases in the discharge pulse. The additional information from the THz TDS diagnostics suggests that towards the end of the pulse the plasma in front of the target is dominated by the singly charged ions of the sputtered material. The influence of the peak discharge current and the pressure of the background gas are also discussed. 

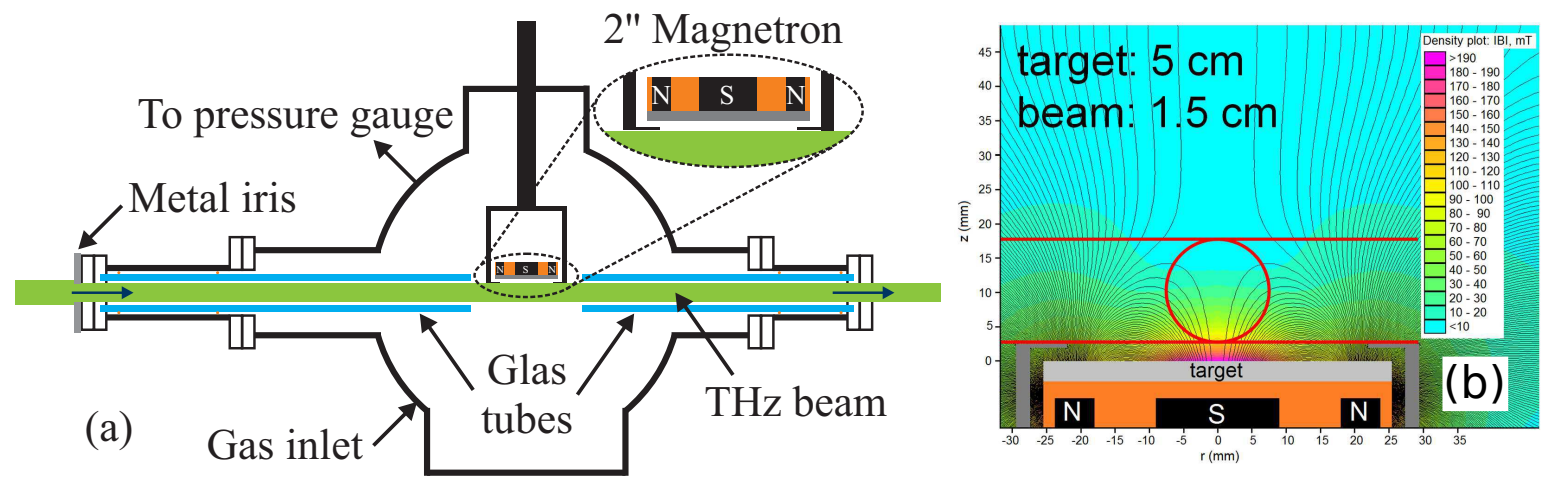

Figure 1. (a) Schematic representation of the discharge chamber with the magnetron and the arrangements to prevent coating of the windows and ensuring good transmissivity for the THz beam. (b) Enlarged view of the magnetron with the distribution of the magnetic field and the volume through which the $\mathrm{THz}$ beam was passing. The circle represents the $\mathrm{THz}$ beam seen along its direction of propagation.

\section{Experimental arrangements}

\subsection{Discharge chamber}

The measurements were performed for a $2^{\prime \prime}$ (5 cm diameter) circular planar magnetron with a titanium target and argon as the background gas. The discharge was ignited in a spherical chamber with an outer diameter of $25 \mathrm{~cm}$ (figure 1(a)). The magnetron was mounted from above into the chamber in a way, that the vertical position of the magnetron could be easily varied. This was used to adjust the position of the magnetron relative to the $\mathrm{THz}$ beam (see below). The configuration and the strength of the magnetic field of the magnetron is shown in figure 1(b). The pressure of the background gas was set to $0.5 \mathrm{~Pa}$ and $2 \mathrm{~Pa}$ and was monitored independently by two gauges (Pfeiffer vacuum, PKR251 and MKS Instruments, 127AAX-000010S).

The discharge was ignited by supplying power from a HiPIMS power supply (SPIK1000A, MELEC GmbH). The power supply was connected to the magnetron through a home made inductive-resistive (LR) matchbox (more details in [26]). The power supply was operated in voltage control mode. The LR matchbox was used as a passive way to control the current rise by reducing the voltage after the breakdown and to achieve a current steady-state condition. The power supply provided a voltage pulse with a length of $200 \mu \mathrm{s}$ and a repetition frequency of $25.3 \mathrm{~Hz}$. The latter value was determined by the $\mathrm{THz}$ setup (see section 2.3). The power was varied by adjusting the output voltage to obtain the desired peak discharge current. The current was monitored using a current probe (Tektronix A622, bandwidth $100 \mathrm{kHz}$ ) and the voltage was measured using a high-voltage probe (Tektronix P6015A, bandwidth $75 \mathrm{MHz}$ ). The presented measurements are for peak currents of $20 \mathrm{~A}, 40 \mathrm{~A}, 60 \mathrm{~A}$, and $80 \mathrm{~A}$. These values correspond to peak discharge current densities (averaged over the magnetron surface) of $1 \mathrm{~A} / \mathrm{cm}^{2}, 2 \mathrm{~A} / \mathrm{cm}^{2}, 3 \mathrm{~A} / \mathrm{cm}^{2}$, and $4 \mathrm{~A} / \mathrm{cm}^{2}$, respectively. 


\subsection{Optical emission measurements}

For recording the temporal evolution of the emission intensities a compact spectrometer (Avantes AvaSpec-ULS2048XL) was used. The spectrometer had two channels that cover the wavelength range between 200 and $1100 \mathrm{~nm}$. The sensitivity of the spectrometer was relatively calibrated and had a full width at half maximum (FWHM) resolution of $0.75 \mathrm{~nm}$. The emission intensity was recorded temporally resolved with an integration time of $5 \mu \mathrm{s}$. Each temporal trace was accumulated 10 times. This resulted in a standard deviation of about $3 \%$. Due to the difference in the emission intensity from the various lines, they were scaled (divided by a factor $\alpha$ ) for ease of the presentation. The scaling factors $\alpha$ are listed in table 1. The recorded emission lines originated from the atoms and the singly charged ions of argon (Ar I and Ar II) as well as from the atoms and the singly and doubly charged ions of titanium (Ti I, Ti II and Ti III). Emission from these species has been chosen, as it is expected that these are dominant constituents in the discharge. Table 1 contains a list of the measured spectral lines, together with some characteristic parameters of the transitions and the plasma species from which the emission originates. All lines are characterized by large values of their Einstein coefficients. Consequently, the lifetimes of the excited species are of the order of ns or shorter if quenching is also considered. These lifetimes are much shorter than the temporal resolution of the spectrometer. Therefore, the measured intensities are representative of the instantaneous densities of the excited species. Under conditions of direct excitation from the ground state the intensities are also representative for the densities of the respective ionic and atomic species. However, contribution by cascade and stepwise processes and variation in time of the electron density and mean energy can influence the relation between emission intensities and densities of the species. To avoid this ambiguity, an elaborate time-dependent collisional-radiative model that includes the majority of the excited species will be needed. However, the intention of showing the emission intensities here is not to develop such a model but rather to establish a connection with other investigations where the emission intensities offer the only diagnostic information about the discharge.

The emission was collected along the same line of sight as the $\mathrm{THz}$ beam path by using an optical fiber. The optical access was through one of the two z-cut quartz windows positioned in opposite flanges. To avoid coating of the windows additional provisions were made to guarantee a permanent optical access. This was especially crucial for the $\mathrm{THz}$ diagnostics, where long-term stability was required. As a first measure the windows were moved as far away from the discharge as possible by increasing the length of the flanges. Here a limiting factor for the maximal distance was the $\mathrm{THz}$ setup (see below). However, this was not sufficient and as a second measure glass tubes were inserted from both sides into the flanges. The tubes were positioned directly in front of the window and ended at a distance of about $1.5 \mathrm{~cm}$ from the magnetron (figure 1(a)). The glass tubes had an outer diameter of $2.5 \mathrm{~cm}$ and an inner diameter of $2 \mathrm{~cm}$. The increased distance between the windows and the magnetron and the small 
Table 1. List of the spectral lines from the different species whose temporal evolution has been measured. The wavelength, $\lambda$, the transition probability, $A_{u \rightarrow l}$, and the energies of the upper, $E_{u}$, and the lower, $E_{l}$, level of the transition are given together with the ionization energies of the corresponding species, $E_{i}$. The energies are relative to the ground state of the corresponding atom or ion. Figure 2 gives a graphical representation of the energy levels. The data are taken from [27]. The last column lists the scaling factors, $\alpha$, by which the intensities have been divided for convenience of presentation. A larger factor corresponds to stronger lines.

\begin{tabular}{lccrrrr}
\hline Species & $\lambda[\mathrm{nm}]$ & $A_{u \rightarrow l}\left[10^{7} \mathrm{~s}^{-1}\right]$ & $E_{l}[\mathrm{eV}]$ & $E_{u}[\mathrm{eV}]$ & $E_{i}[\mathrm{eV}]$ & \multicolumn{1}{c}{$\alpha$} \\
\hline Ar I & 912.30 & 1.89 & 11.55 & 12.91 & 15.78 & 80 \\
Ar II & 434.81 & 11.71 & 16.64 & 19.49 & 27.63 & 30 \\
Ti I & 399.86 & 4.81 & 0.05 & 3.15 & 6.83 & 9 \\
Ti II & 334.90 & 16.1 & 0.61 & 4.31 & 13.58 & 750 \\
Ti II & 334.94 & 16.8 & 0.05 & 3.75 & 13.58 & 750 \\
Ti III & 298.47 & 19 & 5.17 & 9.32 & 27.49 & 35 \\
\hline
\end{tabular}

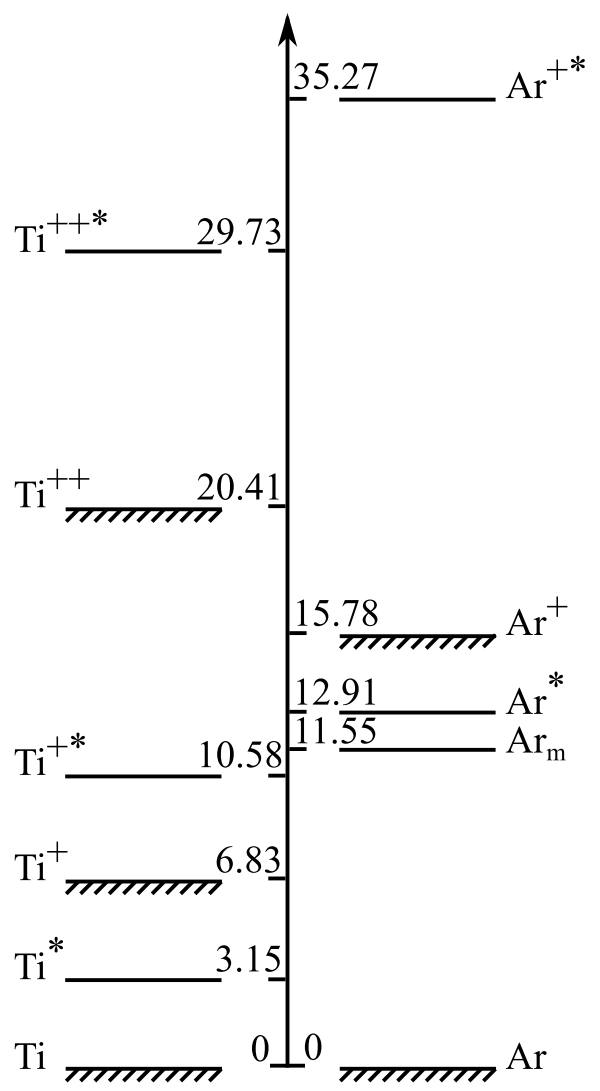

Figure 2. Energy level scheme of the titanium atom including the first two ionization stages and of the argon atom with its first ionization stage. The upper levels of the measured emission lines together with the lower metastable state of the argon atom are also shown. The energies (from the ground state of the respective atomic species) are given in $\mathrm{eV}$. 
aspect ratio of the glass tubes provided a major decrease of the flux of coating particles to the windows. This ensured constant transmissivity of the windows during the course of the measurements.

\subsection{THz time domain spectroscopy}

The non-invasive character of the THz TDS spectroscopy and its good sensitivity for plasma densities in the range typical for HiPIMS discharges $\left(10^{12} \mathrm{~cm}^{-3}\right)$ make this technique ideal for measuring the density evolution in the ionization region. The technique shares some similarities with microwave interferometry and offers good spatial and temporal resolution. The spatial resolution here was limited by the size of the beam to $1.5 \mathrm{~cm}$. The temporal resolution could potentially reach few hundred ps [28]. However, this entails longer measurement times that impose stringent constrains on the stability and the reproducibility of the discharge. In this work a much lower resolution of $3.85 \mu$ s was chosen as a compromise between measurement time and ability to resolve important time scales. Still, slight fluctuations in the discharge shot-to-shot operation and long-term stability manifested themselves as oscillations and uncertainties in the measured electron density.

The $\mathrm{THz}$ system used in this work was based on the newly developed method of dual-frequency multichannel boxcar $\mathrm{THz}$ time domain spectroscopy. The method is described in greater detail elsewhere [28] and here only a brief overview is given. Figure 3 shows a schematic representation of the setup of the $\mathrm{THz}$ system. The $\mathrm{THz}$ pulses were produced by a semiconductor (LT-GaAs) dipole antenna (transmitter module) with a bow-tie electrode configuration (electrode distance of $50 \mu \mathrm{m}$ ). The gap between the electrodes was irradiated by a Ti:sapphire fs-laser (Coherent MIRA, pulse length $176 \mathrm{fs,}$ repetition frequency $76 \mathrm{MHz}$, pulse energy $0.4 \mathrm{~nJ}$, central wavelength $790 \mathrm{~nm}$ ). The laser radiation produced free charge carriers in the semiconductor that were accelerated by a voltage applied to the electrodes of the bow-tie antenna. The voltage had a sinusoidal waveform with a frequency of $38 \mathrm{MHz}$ (half of the laser repetition frequency). The short current pulses in the emitter produced ps-long electromagnetic pulses with the spectrum in the $\mathrm{THz}$ range $(0.1-3 \mathrm{THz})$.

The detection of these pulses was realized by an electro-optical detection. Each $\mathrm{THz}$ pulse was overlapped with a second fs-laser pulse in an electro-optical crystal (ZnTe). The electric field of the $\mathrm{THz}$ pulse changed the refractive index of the crystal (Pockels effect) which then altered the polarization of the laser beam. The difference between the two linear polarization components was proportional to the strength of the $\mathrm{THz}$ electric field. The polarization components of the laser beam were separated by a polarizing beam splitter and were measured by a balanced photo-detector (Thorlabs, PDB430A-AC, bandwidth $350 \mathrm{MHz}$ ). The difference signal from the balanced detector was fed to a lock-in amplifier with integrated multichannel boxcar option (UHFLI, Zurich Instruments). The boxcar averager of the lock-in amplifier was triggered by the sinusoidal voltage applied to the bow-tie antenna. 


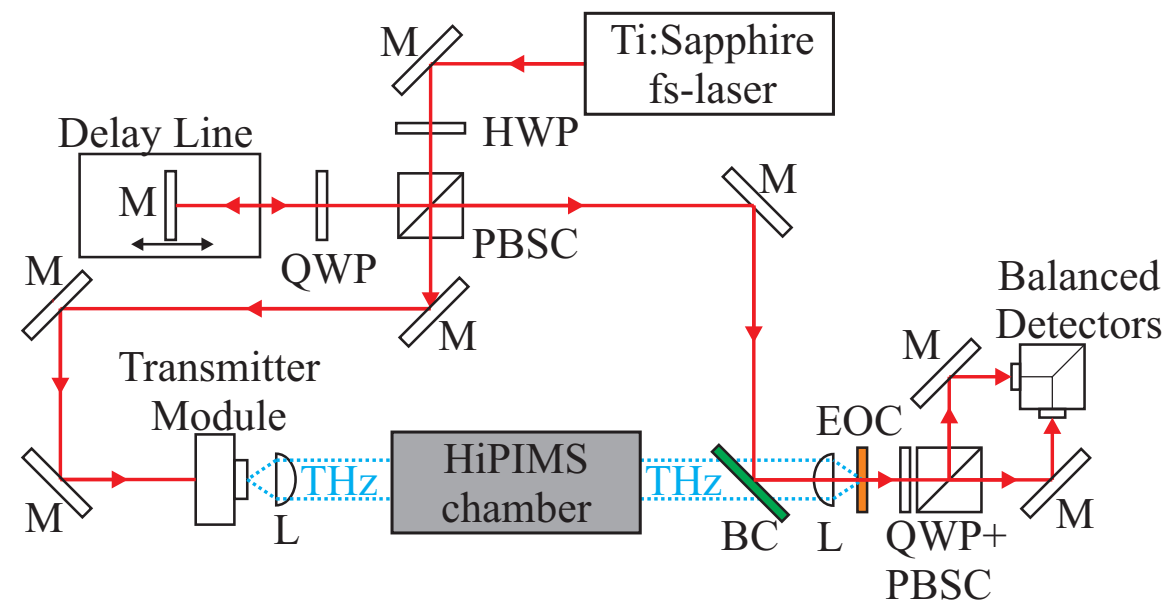

Figure 3. Schematic representation of the THz setup. The abbreviations stand for the following: $\mathrm{M}$ - mirror; HWP - half-wave plate; QWP - quarter-wave plate; PBSC polarization beam splitter cube; L - lens; BC - beam combiner; EOC - electro-optical crystal.

Since the length of the laser pulses was much shorter than the length of the $\mathrm{THz}$ pulses, the detection gave the $\mathrm{THz}$ electric field only for a specific moment in time. To record the $\mathrm{THz}$ electric field at different times of the pulse the second fs-laser beam was delayed by a mechanical delay line. In this way the temporal evolution of the $\mathrm{THz}$ electric field was probed stroboscopically.

The discharge was pulsed synchronously with the laser pulses, i.e. also with the $\mathrm{THz}$ pulses. The pulse frequency for the discharge was $25.3 \mathrm{~Hz}$. This frequency was obtained from a fast delay generator (Stanford Research Systems, DG645, 25 ps rms jitter) by dividing the frequency of the bias voltage on the bow-tie antenna by a factor of 1500000 . The pulsing frequency of the discharge triggered also the periodic waveform analyzer (PWA) of the lock-in amplifier. From the pulse period $(\approx 39.5 \mathrm{~ms})$ a region of interest was selected and internally divided into 1024 equally long temporal channels. This determined the temporal resolution of $3.85 \mu \mathrm{s}$. The boxcar averager of the lockin amplifier detected single events and sorted every event into the corresponding time channel of the PWA. When all channels were filled with the preset number of events $\left(8.4 \cdot 10^{6}\right)$ a signal was sent to move the delay line to the next position. Due to the dead time of the lock-in amplifier, not all channels were filled equally fast. This had the side effect that the measurement time was somewhat longer than initially estimated when some channels did not get sufficient events. This procedure allowed the electric field for the $\mathrm{THz}$ pulses passing through the discharge at 1024 different time instants within the region of interest to be obtained simultaneously. Under these settings a measurement at a single discharge condition took about 3 hours. Therefore, the results shown here are an average over about a quarter of a million discharge periods.

To avoid unwanted reflections from the metal chamber, the diameter of the $\mathrm{THz}$ beam was limited through metal iris apertures, which were placed directly in front of the entrance window on the outside. A compromise between signal strength and spatial 
resolution was achieved through a beam diameter of $1.5 \mathrm{~cm}$. To determine the best height for the magnetron, the $\mathrm{THz}$ signal strength was monitored while the height was altered. The best position was determined by a maximum in the $\mathrm{THz}$ signal strength in a combination with the lowest position of the magnetron. The resulting distance between the edge of the $\mathrm{THz}$ beam and the surface of the target was $2 \mathrm{~mm}$ (about 10 $\mathrm{mm}$ from the center of the beam). In this way it was ensured that the $\mathrm{THz}$ beam was as close as possible to the surface of the magnetron. Furthermore, at this position the complete $\mathrm{THz}$ beam was passing through the region of magnetic field lines that were closing on the magnetron surface (figure 1(b)), i.e. it was passing through the center of the plasma.

The method for obtaining the electron density from the measured $\mathrm{THz}$ pulses is described in greater detail elsewhere [28] and here only a brief overview is given. A Fourier transform is used to determine the amplitude and the phase of the different frequency components in the measured pulses. The density is obtained from the frequency dependence of the phase. Due to the presence of the plasma, the phases of the different frequencies are changed by an amount dictated by the complex dispersion relation of electromagnetic waves in plasma. Essentially for all laboratory plasmas and for electromagnetic pulses with a spectrum in the $\mathrm{THz}$ range a static magnetic field has a negligible effect since even in a magnetic field of $1 \mathrm{~T}$ the electron cyclotron frequency is well below the lowest frequency in the THz pulse [28]. Therefore, here the phase shift as a function of the frequency is fitted to the usual dispersion relation for non-magnetized plasmas with the electron plasma frequency as a fitting parameter. The electron density is then calculated from the fitted value of the plasma frequency.

\section{Results and discussions}

First the temporal evolution of the electron density for the four values of the peak discharge current at an argon pressure of $0.5 \mathrm{~Pa}$ is presented. This evolution is correlated with the discharge voltage and current. For the better understanding of the processes occurring in the discharge and for providing a reference to other studies on the topic, also the temporal evolution of the intensities of selected spectral lines emitted by various atomic and ionic species are shown and discussed. Finally, the measurements at a pressure of $2 \mathrm{~Pa}$ and a discharge current of $60 \mathrm{~A}$ are presented and used to show the influence of the pressure.

\subsection{Temporal evolution of the plasma density}

Figures $4-7$ show the temporal evolution of the various quantities during and shortly after the discharge pulse at an argon filling pressure of $0.5 \mathrm{~Pa}$ : discharge voltage $U_{\mathrm{d}}$, discharge current $I_{\mathrm{d}}$, electron density $n_{\mathrm{e}}$ and intensities of the chosen atomic and ionic lines $I_{x}$, where $x$ stands for the wavelength in nm (table 1 ). The zero of the time axis is shifted to the instant of the voltage rise. However, the trigger pulse arrives about 
$3.8 \mu$ s earlier. This delay is consistent throughout all the measurements and is caused by the electronics of the power supply. The pulse duration is $200 \mu$ s, i.e., the voltage is switched off at $200 \mu \mathrm{s}$. To guide the eye, vertical lines have been drawn at these time instants. Note further, that all presented voltages are negative, as usual. However, to avoid confusion about the terms "increase" and "decrease", here only the absolute value of the voltage is discussed and plotted in the graphs.

The first notable feature in the figures is the value of the measured electron density. It is of the order of several $10^{12} \mathrm{~cm}^{-3}$ up to few $10^{13} \mathrm{~cm}^{-3}$. The measured line-integrated density has been converted into volumetric density by assuming a homogeneous plasma over the diameter of the magnetron $(5 \mathrm{~cm})$. The obtained values agree well with Langmuir probe measurements carried out further away from the target [10-14]. Close to the target one would expect somewhat higher densities. However, the presented values are line of sight averages over the hot and dense plasma zone and the cold region surrounding it. Results from electrical [17,29] and optical emission [30-32] measurements indicate that most of the electrons are confined within the closed magnetic field region in the target vicinity (figure 1(b)). Their numbers are falling sharply outside the confinement region. This is also in good agreement with simulation results [33]. Therefore, the given values here are probably lower than the peak density in the ionization region but nevertheless fit well with the expected order of magnitude.

Another thing to note is the fact that the obtained plasma densities are close to the lower detection limit of the $\mathrm{THz}$ system. The relative uncertainties are then probably larger than expected. One has to also consider that the long integration times and the stability of the discharge are probably also contributing to this. Consequently, it is difficult to discuss details in the behaviour of the electron density during the discharge pulse. Nevertheless, the general trends are well recognizable. The measured values indicate a significant ionization degree (the ratio of the plasma density to the net particle density). The density of the neutral atoms at the filling pressure of $0.5 \mathrm{~Pa}$ is $1.2 \times 10^{14}$ $\mathrm{cm}^{-3}$. Then already at the lowest discharge current of $20 \mathrm{~A}$ the ionization degree exceeds $3 \%$. At the highest discharge current, i.e. the case with the highest electron density, the ionization degree reaches $9 \%$. The actual values are probably even higher than these estimates, since gas rarefaction effects $[34,35]$ due to gas heating and plasma pressure can reduce the density of the neutral atoms. For instance, it is known that the gas temperature during a HiPIMS pulse can exceed $1000 \mathrm{~K}$ [36].

\subsection{Phases of the discharge}

The next notable feature is the existence of several distinct phases in the pulses. These phases have been described previously (see, e.g., [37] and [3, pages 29-31] for an overview). We have marked them in figures 4-7 with the labels I to IV for convenience. Note that we use slightly different names for these phases that describe better the processes under our conditions. The phases are termed here as pre-breakdown (phase I), argon sputtering (phase II), transition (phase III) and self-sputtering (phase IV) 


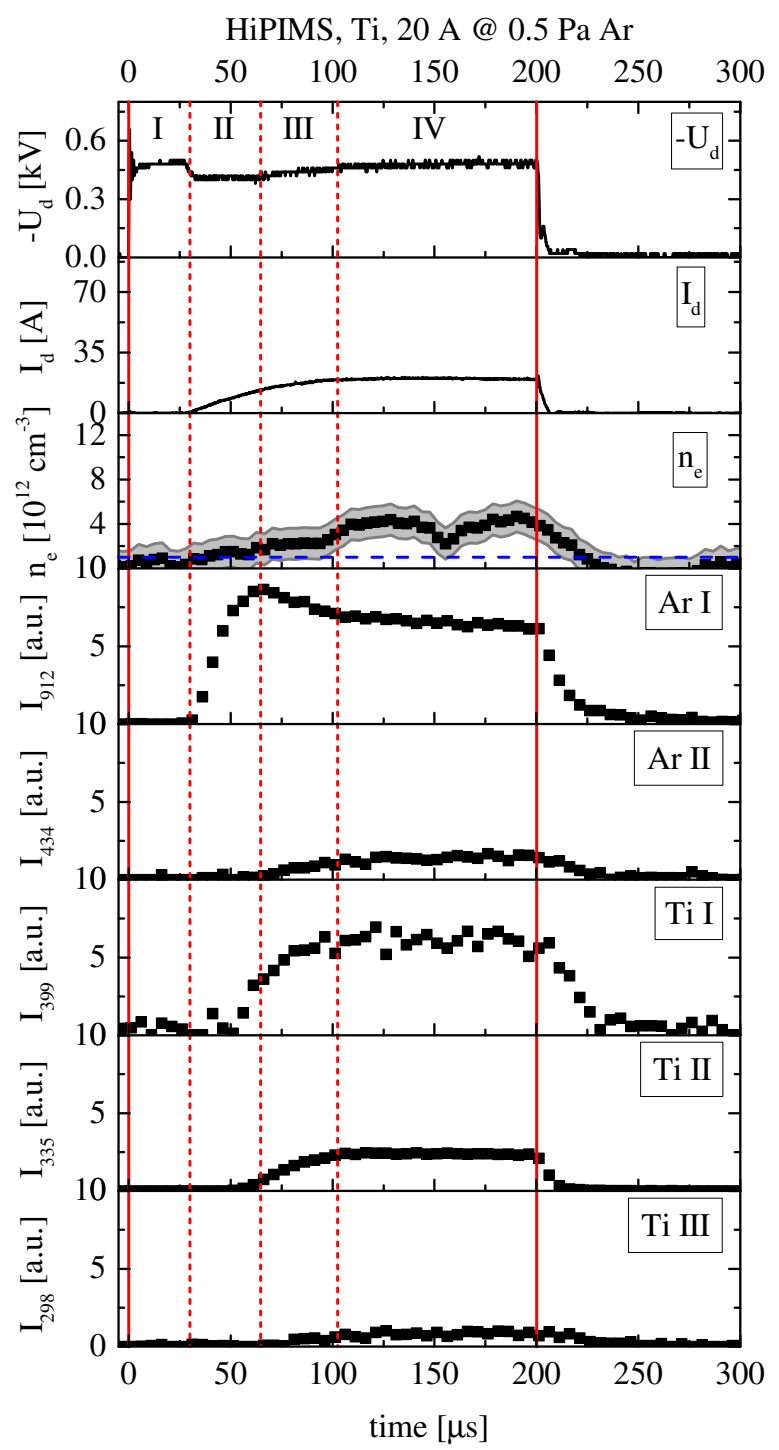

Figure 4. Temporal evolution of the discharge voltage $U_{\mathrm{d}}$, the discharge current $I_{\mathrm{d}}$, the plasma density $n_{\mathrm{e}}$ and the intensities of the selected emission lines for a peak current of $20 \mathrm{~A}$ and a pressure of $0.5 \mathrm{~Pa}$. For clarity of the presentation the negative discharge voltage has been inverted. The grey area in the density plot gives the uncertainty of the measurements and the dashed horizontal line shows the detection limit of the $\mathrm{THz}$ diagnostics $\left(1 \times 10^{12} \mathrm{~cm}^{-3}\right)$. Vertical continuous lines indicate the beginning and the end of the voltage pulse and vertical dashed lines mark the different phases during the discharge pulse.

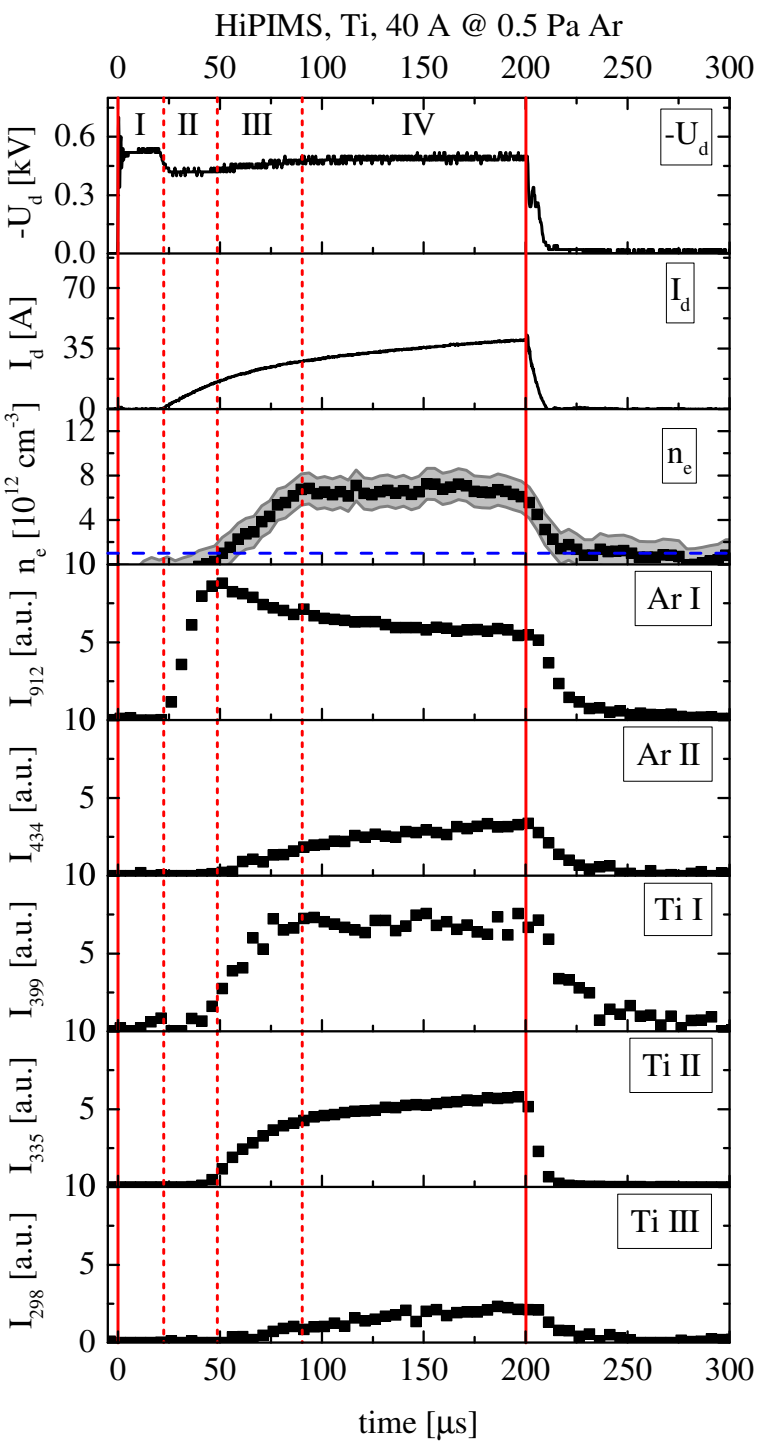

Figure 5. The same as in figure 4 for a peak current of $40 \mathrm{~A}$. 


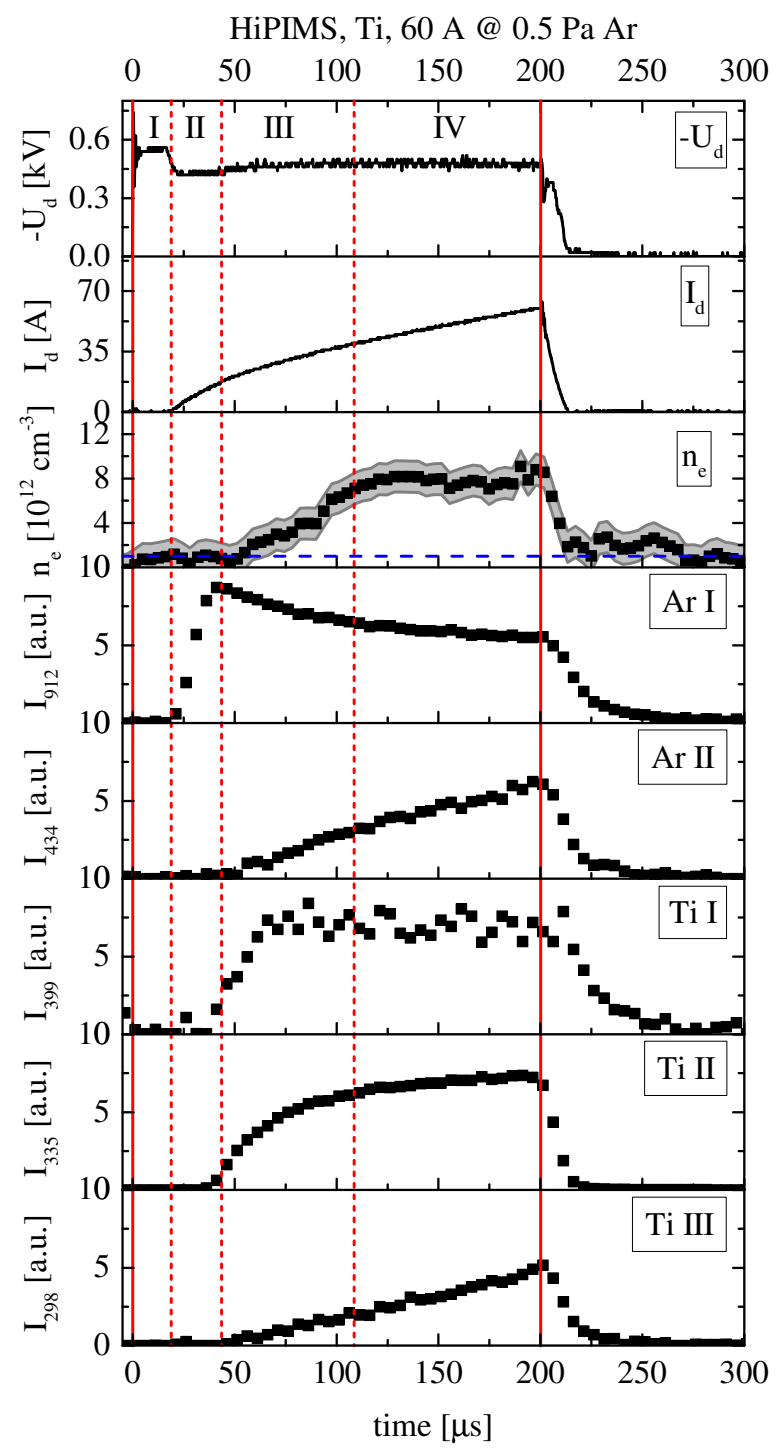

Figure 6. The same as in figure 4 for a peak current of $60 \mathrm{~A}$.

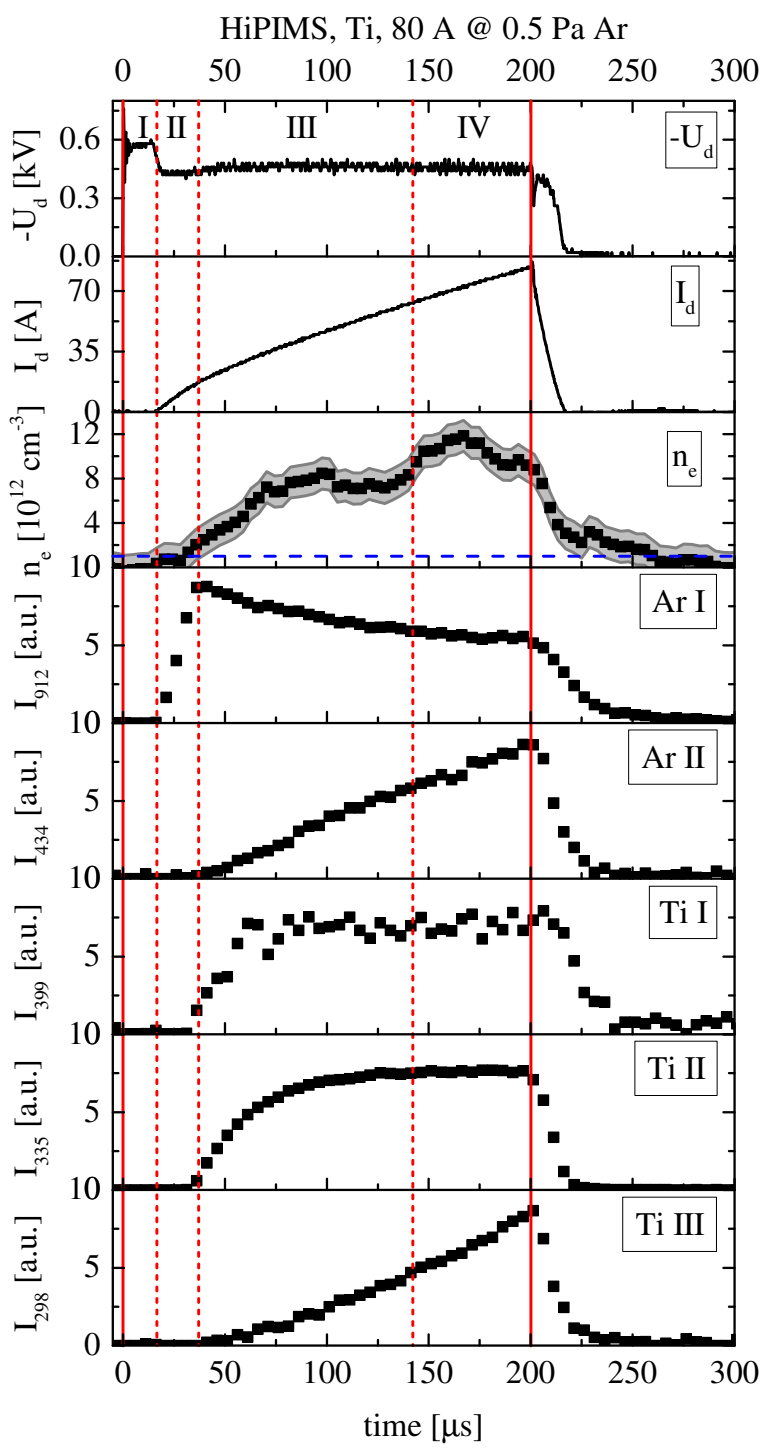

Figure 7. The same as in figure 4 for a peak current of $80 \mathrm{~A}$.

phase. The novelty is the additional information that the temporal evolution of the electron density brings to the description of the discharge behaviour.

During the initial pre-breakdown period (phase I) the applied voltage is nearly constant in time but no considerable current flows and the electron density remains below the detection limit of the $\mathrm{THz}$ system. Also no emission is visible. The duration of this phase decreases with the increase in the peak discharge current. The physical reason for the decrease in the duration is actually the increase in the voltage. Since this is not well visible in the figures, the values are listed in table 2 . This initial phase is the so called formative delay [38]. It is known and well understood in HiPIMS discharges. For example, it has been shown to depend on the pressure [11] as well as on the discharge voltage and the secondary electron emission coefficient from ion bombardment of the target material [39]. Similar pre-breakdown delays are known also for other types of 
Table 2. The voltage during the pre-breakdown phase, $U_{I}$, and the duration of that phase, $\Delta t_{I}$ as a function of the peak discharge current, $I_{\mathrm{d}}$.

\begin{tabular}{lll}
\hline$I_{\mathrm{d}}[\mathrm{A}]$ & $U_{I}[\mathrm{~V}]$ & $\Delta t_{I}[\mu \mathrm{s}]$ \\
\hline 20 & 480 & 30.0 \\
40 & 520 & 21.0 \\
60 & 560 & 18.4 \\
80 & 580 & 16.2 \\
\hline
\end{tabular}

discharges, e.g. atmospheric pressure ns-pulsed discharges in helium [40,41].

Simply put, the discharge ignition requires that the initial seed electrons are generated and multiplied to a certain critical level. The responsible mechanisms for that might vary, e.g., a Townsend avalanche or secondary electrons emitted from the target in the case of HiPIMS discharges [39]. In all cases the critical electron density, that needs to be attained, has to be sufficiently high to start influencing the external electric field [38]. This allows the build up of the high voltage sheath in front of the target and the transition to the discharge phase.

When the breakdown occurs, the discharge enters the argon sputtering phase (phase II). The current and the density start to rise and the voltage drops to $420 \mathrm{~V}$ (for the $0.5 \mathrm{~Pa}$ case). This effect is commonly observed when the power level from the supply is kept constant: as the discharge impedance decreases at breakdown, the voltage has to be reduced to keep the power constant. However, in our case this is due to the LR matchbox, that is reducing the voltage as the current rise occurs in order to optimize the current rise time. During this phase the only emission comes from the excited argon atoms. Notably no emission from particles of the target material is detected. Since the energy levels of titanium lie below the levels of argon (cf figure 2), this indicates that during this phase only the background gas is excited and ionized while the electron density builds up. Initially the sputtering is not significant and is caused by argon atoms. Consequently, no atoms of the target material are present in the plasma, which explains the lack of titanium emission. Once the plasma density is sufficiently high, the sputtering of the titanium target becomes efficient. Further, it has to be noted that comparing figures $4-7$ it is evident that the duration of this second phase decreases with the increase in the peak discharge current. This is again a consequence of the LR matchbox - with higher peak current the matchbox allows for faster current increase. This leads to faster plasma density buildup and results in an earlier initiation of the sputtering of the target material.

The appearance of the first emission from excited titanium atoms marks the transition of the discharge pulse into phase III. During this phase the discharge voltage starts to increase slightly and the discharge current changes its slope. Further, emission from ionic species (Ar II, Ti II and Ti III) appears with the onset of this phase. Only the intensity of the atomic argon lines starts to decrease as reported also in a recent publication by Fekete et al [42]. This forms a maximum in the emission from the argon 
atoms. Actually, this maximum has been used here to define the transition between phase II and III, since its occurrence is better defined. The measured electron density is in the range of a few $10^{12} \mathrm{~cm}^{-3}$ and continues to increase during this phase. The duration of this phase varies with the peak discharge current. It steadily increases with the increase in the peak current value.

These trends reveal the following picture. The plasma created in phase II has initiated the sputtering process. Atoms of the target material are now injected into the plasma zone where they are immediately ionized by electron impact. The titanium atoms can also quench the excited argon atoms, further leading to the ionization of the sputtered target material (Penning process). The argon ions can ionize not only the titanium atoms but also the singly charged titanium ions (figure 2). However, the ionization energy of the argon ions is not sufficient for double ionization of the titanium atoms. As a result of the quenching processes during this phase, the majority of the excited argon atoms and argon ions are destroyed by the incoming sputtered titanium atoms. This has been observed previously for the argon metastable atoms [37]. The comparable masses of titanium and argon allows the wind of sputtered titanium material to also expel the argon particles from the region above the target [43]. Both effects quenching and sputter wind - lead to the removal of excited argon species from the region above the target. Consequently, the argon emission above the target is extinguished. This has been observed in the Abel inverted images of the emission from excited argon atoms [30,31]. It was shown that towards the end of the discharge pulse the emission from argon atoms originates only from the outer regions of the closed magnetic field region. Since the emission measurements here are line integrated, this explains the observed reduction in the intensity of the atomic argon lines.

The net result is that during this transition phase the ionization gradually shifts from ionization of the background gas to ionization of the sputtered titanium. Further, the sputtering shifts from sputtering by the argon ions to self sputtering by the titanium ions. Naturally, at higher peak current values a larger amount of argon ions needs to be replaced by titanium ones. This requires more material to be sputtered, leading to the longer duration of the transition phase.

The transition is related also to a change in the balance of secondary electrons. These electrons give probably the largest contribution to the ionization. During phase II the surface is bombarded by argon ions only. Their secondary electron yield on titanium is relatively large and the ionization is easily sustained by the influx of secondary electrons. However, as the ion composition gradually shifts from argon ions towards titanium ions the situation changes. The singly charged titanium ions are characterized by a negligible secondary electron yield. Therefore, argon ions or doubly charged titanium ions are needed to sustain the influx of secondary electrons. However, the production of such ions requires more energy. Consequently, the discharge voltage has to increase, increasing also the energy of the secondary electrons. The latter is observable in the appearance of emission of ionic lines from $\mathrm{Ar}^{+}$and $\mathrm{Ti}^{++}$ions.

The last phase of the pulse, phase $I V$, is marked by a nearly constant voltage and 
a linear increase in the current. The only exception is the $20 \mathrm{~A}$ case where the current saturates at this value. This phase is further marked by constant emission intensity of the atomic lines of argon and titanium. The intensity of the Ti II line shows also a saturation-like behaviour. Notably also the electron density exhibits no significant variation and remains essentially constant.

This phase is known also as the runaway phase - due to the high power density, the phase is dominated by the self-sputtering of titanium [3] as is evident also from the results. The constant electron density correlates well with the constant intensity of the emission from $\mathrm{Ti}^{+}$ions. Since the emission from the other ionic species $\left(\mathrm{Ar}^{+}\right.$and $\mathrm{Ti}^{++}$) continues to rise, one can conclude that the singly charged titanium ions are the dominant ionic species. Recent modeling results [44] are in good agreement with the present picture. This hypothesis is further supported by the relative intensities of the emission lines from the $\mathrm{Ar}^{+}$and the $\mathrm{Ti}^{+}$ions, the latter being more than an order of magnitude stronger (evident from the scaling factor $\alpha$ in table 1). However, it has to be stressed that this is only a hypothesis that would require further investigations to be confirmed or rejected. The recorded emission lines of the $\mathrm{Ar}^{+}$and $\mathrm{Ti}^{++}$ions originate both from states that are relatively high on the energetic scale (cf figure 2). Therefore, it is most likely that these emission intensities indicate a steady increase in the flux of secondary electrons. This means an increase in the density and/or the energy of these electrons. The increase in the flux of secondary electrons drives the increase in the discharge current and vice versa. Simultaneously, the secondary electrons produce the $\mathrm{Ar}^{+}$and $\mathrm{Ti}^{++}$ions. In turn these ions are crucial for the release of electrons from the surface of the target. It has been argued [45] that the presence of argon in the active plasma zone above the target is a result from argon being trapped in the target during the early stages of the HiPIMS pulse. The Ar is sputtered from the target, ionized, and accelerated back to the target, completing a recycle loop. It is also possible that not all argon particles have been expelled from the active region.

After the end of the voltage pulse at $200 \mu$ s the afterglow phase follows. During this phase the current, the voltage and the electron density all decay within few $\mu$ s to zero. It has to be noted that the decay in the current is not caused by the decrease in the plasma density, as the latter decays at much slower rate than the current. This trend is consistent with the measurements by Bohlmark et al [17] and by de Poucques et al [46]. They have shown that this effect is caused by the plasma bulk slowly diffusing away from the target in the afterglow. However, it has to be noted that the plasma density under their conditions is about two orders of magnitude lower than the densities presented here. Therefore, in contrast to the work of, e.g., de Poucques et al [46], the recombination dominates over diffusion. Using the values for the ambipolar diffusion coefficient given in [46] and adjusted for the pressure, a diffusion time of $1.6 \mathrm{~ms}$ is obtained for our conditions, whereas the characteristic time scale for three-body recombination is much shorter. Using data from [47], the recombination time is estimated to be $0.37 \mu \mathrm{s}$.

The dominating recombination process is evident also in the behaviour of the emission intensities. While all ionic lines start to decay with the pulse termination, 


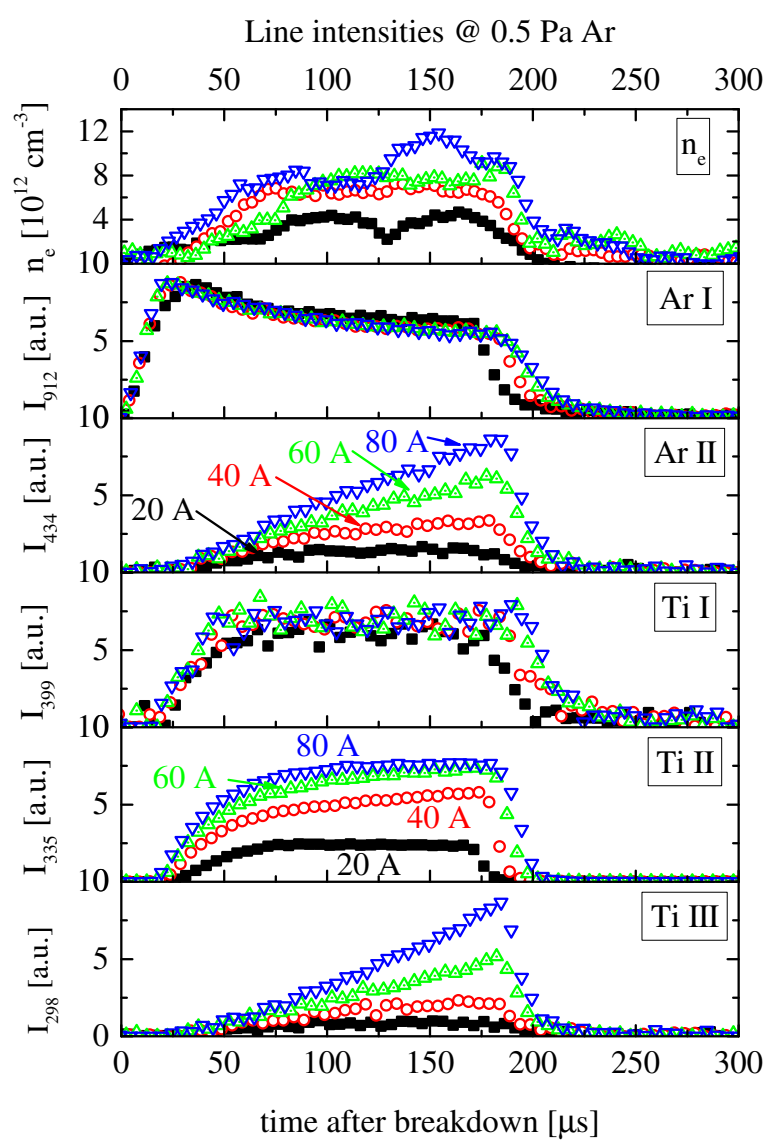

Figure 8. Comparison of the temporal evolution of the electron density and the intensities of the emission lines from Ar I, Ar II, Ti I, Ti II, and Ti III for different peak discharge currents at a pressure of 0.5 Pa. For better comparison the pre-breakdown phase has been removed from each dataset. Consequently, the zero of the time axis represents the moment of the breakdown (the beginning of phase II).

the atomic ones persist for about $10 \mu$ s before they also start to decay. Further, the decay of the atomic lines is slower than the decay of the ionic lines. Both of these are a direct consequence of three-body collisional-radiative recombination, that leads to additional population of the atomic excited states [47,48]. Evidence for population of radiating levels by recombination has been found also by others [49].

\subsection{Correlation with the emission intensities}

To understand the effect of the peak discharge current, the various plasma quantities have been plotted together (figure 8). Note that for better comparison the time axis of each dataset has been shifted so that the breakdown occurs at time zero. This comparison reveals some additional trends.

Not surprisingly, with the increase in the peak discharge current the electron density also increases. However, the increase does not seem to be proportional to the peak current. The emission from the singly charged titanium ions shows similar behaviour 
- it increases with the discharge current, but reaches a saturation. The similarity in the behaviour of the electron density and of the singly charged titanium ions with the discharge current is in conformity with the hypothesis that in the later phases of the discharge pulse the plasma consists mainly of electrons and titanium ions.

Another expected feature is the increase in the emission of the ions that produce the secondary electrons $\left(\mathrm{Ar}^{+}\right.$and $\left.\mathrm{Ti}^{++}\right)$. This increase is nearly linear and follows well the increase in the discharge current. However, this increase in the ion emission intensities seems to contradict the obtained nearly constant values of the electron density during phase IV (figures 4-7). To understand this behaviour one has to consider that the excitation of these lines requires electrons with energies exceeding $30 \mathrm{eV}$ (see figure 2 and table 1). As discussed, the number of electrons with such large energies is about $10 \%$ of the total electron population, i.e., of the order of the uncertainty of the $\mathrm{THz}$ measurements. It is then evident that an increase in the population of energetic electrons will remain largely undetected in the $\mathrm{THz}$ data whereas it will be clearly visible in the emission intensity.

Interestingly enough, the emission from the atomic species (Ar I and Ti I) does not show any dependence on the peak discharge current. For the argon atoms this is understandable. Initially the discharge burns in argon gas and since the pressure and the voltage are the same (see table 2), the plasma parameters are also the same. During the late phases of the voltage pulse, the emission from the argon atoms originates from the regions outside the active zone of the magnetron. The conditions there are less affected by the sputtering. Consequently, the recorded line integrated emission is also not influenced by the discharge current.

For the emission from the titanium atoms one has to note that it is weak (smallest scaling factor $\alpha$ in table 1). This is related to the fact that most of the neutral titanium is quickly ionized. Consequently, the density of excited atomic states is low and in saturated equilibrium. Therefore, the observed emission from the titanium atoms is not depending on the peak discharge current as well.

\subsection{Influence of the pressure}

Finally, the influence of the pressure on the discharge is discussed. For that, measurements at $2 \mathrm{~Pa}$ argon pressure and a peak discharge current of $60 \mathrm{~A}$ have been performed. The results are presented in figure 9. A comparison with figure 6, which presents the results for the $0.5 \mathrm{~Pa}$ case and the same value of $I_{\mathrm{d}}$, reveals the same phases and trends. However, when the pressure is higher there is hardly a delay in the breakdown, i.e., phase I is almost non-existent. Another difference is the lower discharge voltage throughout - at $2 \mathrm{~Pa} U_{\mathrm{d}}$ during phase $\mathrm{I}$ is lower by more than $10 \%\left(U_{\mathrm{d}}=560\right.$ $\mathrm{V}$ at $0.5 \mathrm{~Pa}$ and $U_{\mathrm{d}}=460 \mathrm{~V}$ at $2 \mathrm{~Pa}$ ). During phase II the difference is almost $20 \%$ $\left(U_{\mathrm{d}}=420 \mathrm{~V}\right.$ at $0.5 \mathrm{~Pa}$ and $U_{\mathrm{d}}=340 \mathrm{~V}$ at $\left.2 \mathrm{~Pa}\right)$ and for phase $\mathrm{IV}$, during which the voltage is also constant, the difference is again $10 \%\left(U_{\mathrm{d}}=480 \mathrm{~V}\right.$ at $0.5 \mathrm{~Pa}$ and $U_{\mathrm{d}}=420$ $\mathrm{V}$ at $2 \mathrm{~Pa})$. 


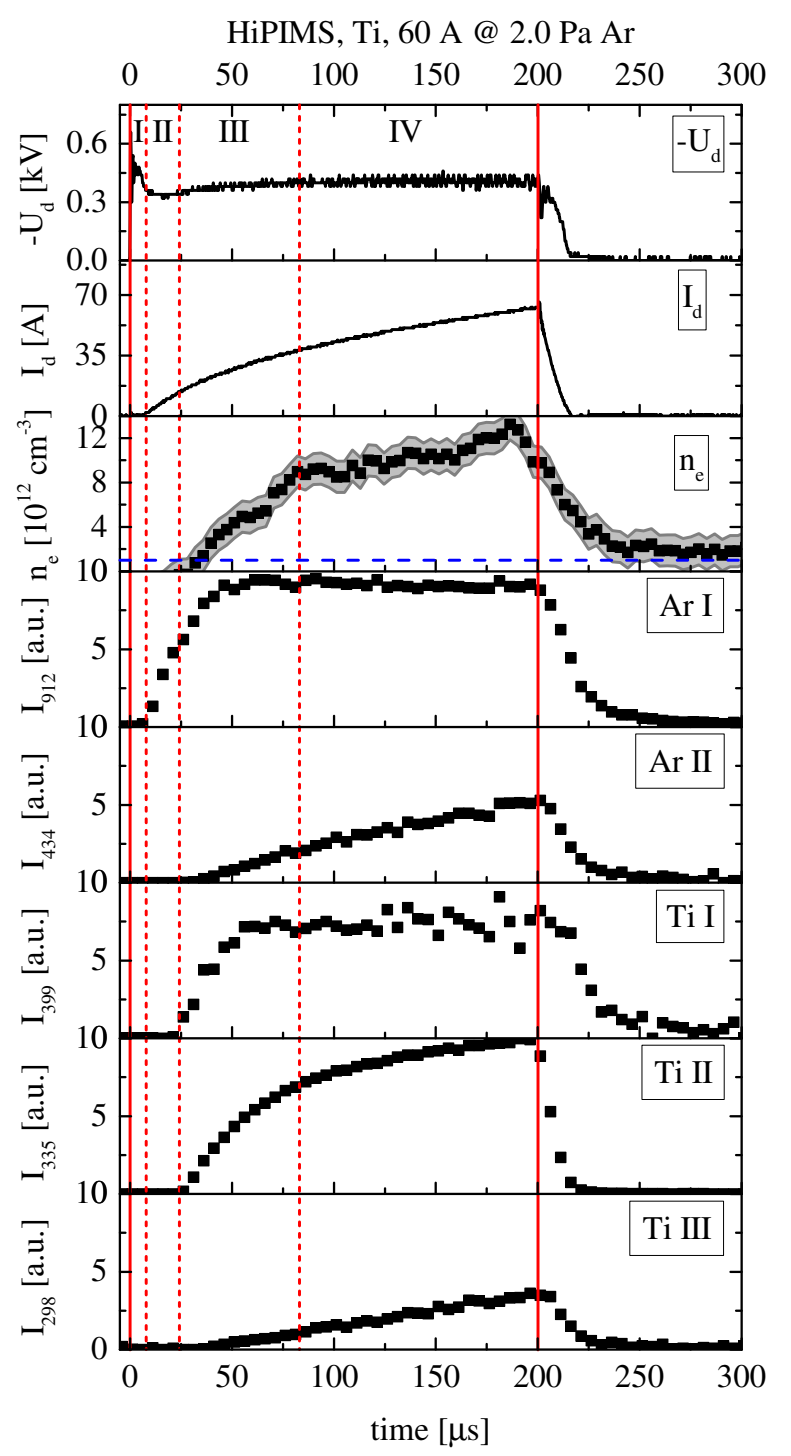

Figure 9. The same as in figure 4 for a peak current of $60 \mathrm{~A}$ and a pressure of $2 \mathrm{~Pa}$.

The importance of these differences are most evident in phase IV. Since the discharge current is the same, a difference in the voltage translates to a difference in the deposited power. Nevertheless, the peak electron density at $2 \mathrm{~Pa}$ is higher by more than $50 \%$ (cf figure 6 and 9). This can be explained by the characteristic energy, $\Theta$, required to produce an electron-ion pair $[50,51]$. With increasing pressure the ionization events become more probable, while the losses of particles are reduced (while still keeping the electrons magnetized). This leads to a reduction in $\Theta$ as the pressure increases. Consequently, higher numbers of electrons and ions can be produced with the same amount of energy deposited in the plasma. 


\section{Conclusions}

Using the novel method of THz time domain spectroscopy a HiPIMS discharge with a titanium target has been investigated. First direct measurements of the electron density in the active zone of the magnetron have been obtained. The determined values of the density are in the range of $10^{12}-10^{13} \mathrm{~cm}^{-3}$ for peak discharge currents in the range of 20 to $80 \mathrm{~A}$. The temporal evolution of the electron density has been correlated with the emission intensity from the various atomic and ionic species present in the discharge. The well known discharge phases have been observed. Most importantly, the measured electron density is nearly constant during the runaway/self sputtering phase. Based on the measurements it has been suggested that during this phase the titanium ions are the dominant heavy particle species. The same trends have been observed also when the pressure was varied.

\section{Acknowledgments}

This work has been supported by the German Science Foundation (DFG) within the frame of the collaborative research centre SFB-TR 87 and the project Cz73/9-1. Financial support from Ruhr-University Bochum through the Research Department "Plasmas with complex interactions" is also gratefully acknowledged. The authors further acknowledge Dr. Christian Mazl for lending the power supply and Thomas Zierow for expert technical support.

\section{References}

[1] Sarakinos K, Alami J and Konstantinidis S 2010 Surf. Coat. Technol. 2041661 - 1684 ISSN 02578972 URL http://www. sciencedirect.com/science/article/pii/S0257897209009426

[2] Lundin D and Sarakinos K 2012 J. Mat. Research 27 780-792

[3] Gudmundsson J T, Brenning N, Lundin D and Helmersson U 2012 J. Vac. Sci. Technol. A 30 030801

[4] Helmersson U, Lattemann M, Bohlmark J, Ehiasarian A P and Gudmunds-

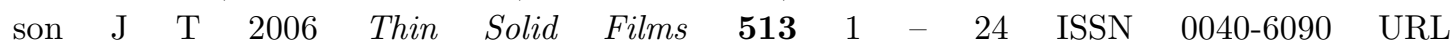
http://www.sciencedirect.com/science/article/pii/S0040609006004317

[5] Anders A 2010 Thin Solid Films 5184087 - 4090 ISSN 0040-6090 URL http://www.sciencedirect.com/science/article/pii/S0040609009018288

[6] Benzeggouta D, Hugon M C and Bretagne J 2009 Plasma Sources Sci. Technol. 18045026

[7] Hala M, Viau N, Zabeida O, Klemberg-Sapieha J E and Martinu L 2010 J. Appl. Phys. 107043305

[8] Vašina P, Meško M, Imbert J C, Ganciu M, Boisse-Laporte C, de Poucques L, Touzeau M, Pagnon D and Bretagne J 2007 Plasma Sources Sci. Technol. 16501 URL http: //stacks . iop.org/0963-0252/16/i=3/a=009

[9] Vašina P, Fekete M, Hnilica J, Klein P, Dosoudilová L, Dvořák P and Navrátil Z 2015 Plasma Sources Sci. Technol. 24065022 URL http://stacks.iop.org/0963-0252/24/i=6/a=065022

[10] Gudmundsson J T, Alami J and Helmersson U 2001 Appl. Phys. Lett. 78 3427-3429

[11] Gudmundsson J T, Alami J and Helmersson U 2002 Surf. Coat. Technol. 161 249-256

[12] Alami J, Gudmundsson J T, Bohlmark J, Birch J and Helmersson U 2005 Plasma Sources Sci. Technol. 14525 
[13] Poolcharuansin P and Bradley J W 2010 Plasma Sources Sci. Technol. 19025010 URL http: //stacks.iop.org/0963-0252/19/i=2/a=025010

[14] Poolcharuansin P, Liebig B and Bradley J 2010 IEEE Trans. Plasma Sci. 38 3007-3015 ISSN 0093-3813

[15] Minea T M, Costin C, Revel A, Lundin D and Caillault L 2014 Surf. Coat. Technol. 25552 61 ISSN 0257-8972 protective Coatings and Thin Films'13 : EMRS 2013 Symposium S URL http://www.sciencedirect.com/science/article/pii/S0257897213011432

[16] Gudmundsson J T, Lundin D, Brenning N, Raadu M A, Huo C and Minea T M 2016 Plasma Sources Sci. Technol. 25065004 URL http://stacks.iop.org/0963-0252/25/i=6/a=065004

[17] Bohlmark J, Gudmundsson J T, Alami J, Latteman M and Helmersson U 2005 IEEE Trans. Plasma Sci. 33 346-347 ISSN 0093-3813

[18] Lockwood Estrin F, Karkari S K and Bradley J W 2017 J. Phys. D: Appl. Phys. 50295201

[19] Hecimovic A, Held J, Schulz-von der Gathen V, Breilmann W, Maszl C and von Keudell A 2017 J. Phys. D: Appl. Phys. 50505204

[20] Hecimovic A, Schulz-von der Gathen V, Böke M, von Keudell A and Winter J 2015 Plasma Sources Sci. Technol. 24045005

[21] Gudmundsson J T, Sigurjonsson P, Larsson P, Lundin D and Helmersson U 2009 J. Appl. Phys. 105123302

[22] Gallian S, Trieschmann J, Mussenbrock T, Brinkmann R P and Hitchon W N G $2015 \mathrm{~J}$. Appl. Phys. 117023305

[23] Corbella C, Marcak A, de los Arcos T and von Keudell A 2016 J. Phys. D: Appl. Phys. 49 16LT01

[24] Lewis M A, Glocker D A and Jorne J 1989 J. Vac. Sci. Technol. A 7 1019-1024

[25] Huo C, Lundin D, Raadu M A, Anders A, Gudmundsson J T and Brenning N 2013 Plasma Sources Sci. Technol. 22045005

[26] Breilmann W, Maszl C, Hecimovic A and von Keudell A 2017 J. Phys. D: Appl. Phys. 50135203

[27] Kramida A, Ralchenko Y, Reader J and and NIST ASD Team 2015 URL http://physics.nist.gov/PhysRefData/ASD/lines_form.html

[28] Meier S M, Tsankov Ts V, Luggenhölscher D and Czarnetzki U 2017 J. Phys. D: Appl. Phys. 50 245202

[29] Lundin D, Sahab S A, Brenning N, Huo C and Helmersson U 2011 Plasma Sources Sci. Technol. 20045003 URL http://stacks.iop.org/0963-0252/20/i=4/a=045003

[30] Liebig B, Braithwaite N St J, Kelly P J and Bradley J W 2010 Thin Solid Films 519 1699-1704

[31] Hecimovic A, de los Arcos T, Schulz-von der Gathen V, Böke M and Winter J 2012 Plasma Sources Sci. Technol. 21035017

[32] Britun N, Palmucci M, Konstantinidis S and Snyders R 2015 J. Appl. Phys. 117163302

[33] Bogaerts A, Bultinck E, Eckert M, Georgieva V, Mao M, Neyts E and Schwaederlé L 2009 Plasma Proc. Polym. 6 295-307 ISSN 1612-8869 URL http://dx.doi.org/10.1002/ppap. 200800207

[34] O'Connell D, Gans T, Crintea D L, Czarnetzki U and Sadeghi N 2008 J. Phys. D: Appl. Phys. 41 035208

[35] Crintea D L, Czarnetzki U, Iordanova S, Koleva I and Luggenhölscher D 2009 J. Phys. D: Appl. Phys. 42045208

[36] Vitelaru C, Lundin D, Stancu G D, Brenning N, Bretagne J and Minea T 2012 Plasma Sources Sci. Technol. 21025010 URL http://stacks.iop.org/0963-0252/21/i=2/a=025010

[37] Kanitz A, Hecimovic A, Böke M and Winter J 2016 J. Phys. D: Appl. Phys. 49125203 URL http://stacks.iop.org/0022-3727/49/i=12/a=125203

[38] Raizer Y P 1997 Gas Discharge Physics 2nd ed (Springer-Verlag) ISBN 3-540-19462-2

[39] Yushkov G Yu and Anders A 2010 IEEE Trans. Plasma Sci. 383028

[40] Schregel C G, Carbone E A D, Luggenhölscher D and Czarnetzki U 2016 Plasma Sources Sci. Technol. 25054003

[41] Carbone E A D, Schregel C G and Czarnetzki U 2016 Plasma Sources Sci. Technol. 25054004

[42] Fekete M, Hnilica J, Vitelaru C, Minea T and Vašina P 2017 J. Phys. D: Appl. Phys. 50365202 
URL http: //stacks . iop.org/0022-3727/50/i=36/a=365202

[43] Kadlec S 2007 Plasma Proc. Polym. 4 S419-S423 ISSN 1612-8869 URL http://dx.doi.org/10.1002/ppap.200731101

[44] Huo C, Lundin D, Gudmundsson J T, Raadu M A, Bradley J W and Brenning N 2017 J. Phys. D: Appl. Phys. 50354003

[45] Anders A, Čapek J, Hála M and Martinu L 2012 J. Phys. D: Appl. Phys. 45012003

[46] de Poucques L, Imbert J C, Boisse-Laporte C, Bretagne J, Ganciu M, TeuléGay L and Touzeau M 2006 Plasma Sources Sci. Technol. $\mathbf{1 5} 661$ URL http://stacks.iop.org/0963-0252/15/i=4/a=010

[47] Tsankov Ts V, Johnsen R and Czarnetzki U 2015 Plasma Sources Sci. Technol. 24065001

[48] Tsankov Ts V, Siepa S, Böhm P S, Luggenhölscher D and Czarnetzki U 2014 IEEE Trans. Plasma Sci. $422388-2389$

[49] Sushkov V, Do H T, Cada M, Hubicka Z and Hippler R 2013 Plasma Sources Sci. Technol. 22 015002 URL http://stacks.iop.org/0963-0252/22/i=1/a=015002

[50] Lieberman M A and Lichtenberg A J 2005 Principles of Plasma Discharges and Materials Processing 2nd ed (Hoboken: Wiley) ISBN 978-0-471-72001-0

[51] Aliev Y M, Schlüter H and Shivarova A 2000 Guided-Wave Produced Plasmas Springer series on Atoms + Plasmas (Berlin: Springer) ISBN 3-540-65273-6 